\title{
Advanced intra-tumoural structural characterisation of hepatocellular carcinoma utilising FDG-PET/CT: a comparative study of radiomics and metabolic features in 3D and 2D
}

\author{
Mohamed Houseni ${ }^{1 A, D, E}$, Menna Allah Mahmoud ${ }^{1 A, B, C D, E, F}$, Salwa Saad ${ }^{2 E}$, Fathi ElHussiny ${ }^{2 A}$, Mohammed Shihab ${ }^{2 D, E}$ \\ 'Department of Medical Imaging, National Liver Institute, Menoufia University, Egypt \\ 2Department of Physics, Faculty of Science, Tanta University, Tanta, Egypt
}

\section{Abstract}

Purpose: The aim of our work is to evaluate the correlation of two-dimensional (2D) and three-dimensional (3D) radiomics and metabolic features of hepatocellular carcinoma (HCC) with tumour diameter, staging, and metabolic tumour volume (MTV).

\begin{abstract}
Material and methods: Thirty-three patients with HCC were studied using ${ }^{18} \mathrm{~F}$-fluorodeoxyglucose positron-emission tomography with computed tomography $\left({ }^{18} \mathrm{~F}\right.$ [FDG] PET/CT). The tumours were segmented from the PET images after CT correction. Metabolic parameters and 35 radiomics features were compared using 2D and 3D modes. The metabolic parameters and tumour morphology were compared using 2 different types of software. Tumour heterogeneity was studied in both metabolic parameters and radiomics features. Finally, the correlation between the metabolic and radiomics features in 3D mode, as well as tumour morphology and staging according to the American Joint Committee on Cancer (AJCC) staging were studied.

Results: Most of the metabolic parameters and radiomics features are statically stable through the 2D and 3D modes. Most of the 3D mode features show a correlation with metabolic parameters; the total lesion glycolysis (TLG) shows the highest correlation, with a Spearman correlation coefficient (rs) of 0.9776. Also, the grey level run length matrix/ run length non-uniformity (GLRLM_RLNU) from radiomics features exhibits a correlation with a Spearman correlation coefficient of 0.9733 . Maximum tumour diameter is correlated with TLG and GLRLM_RLNU, with rs equal to 0.7461 and 0.7143 , respectively. Regarding AJCC staging, some features show a medium but prognostic correlation. In the case of 2D-mode features, all metabolic and radiomics features show no significant correlation with MTV, AJCC staging, and tumour maximum diameter.

Conclusions: Most of the normal metabolic parameters and radiomics features are statistically stable through the 3D and $2 \mathrm{D}$ modes. 3D radiomics features are significantly correlated with tumour volume, maximum diameter, and staging. Conversely, 2D features have negligible correlation with the same parameters. Therefore, 3D mode features are preferable and can accurately evaluate tumour heterogeneity.
\end{abstract}

Key words: hepatocellular carcinoma, FDG-PET-CT, heterogeneity, radiomics.

\section{Introduction}

Hepatocellular carcinoma (HCC) is the most common primary liver malignancy and a leading cause of cancerrelated death [1]. HCC is an extremely heterogeneous disease, and intratumour heterogeneity is a recognised fact within each specific tumour. Heterogeneity could involve molecular, morphological, and immunohistochemical characteristics.

The pathologic classification of HCC is based on the degree of cellular difference. The cancer tissue of two different histological grades may be present in the same

Correspondence address:

Menna Allah Mahmoud, Department of Medical Imaging, National Liver Institute, Menoufia University, Gamal Abdelnasser street, Shebin ElKom, Egypt, e-mail: menna22a@yahoo.com

Authors' contribution:

A Study design - B Data collection - C Statistical analysis - D Data interpretation - E Manuscript preparation - F Literature search - G Funds collection 
tumour. Intratumor heterogeneity of HCC has a prognostic value [2]

It is hard to survey intra-tumoural heterogeneity with conventional testing or biopsy because it is difficult to cover the full degree of phenotypic or hereditary variety inside a tumour. Imaging modalities such as X-ray, ultrasound (US), computed tomography (CT), magnetic resonance imaging (MRI), and positron emission tomography (PET) show a good and non-intrusive technique for evaluating the heterogeneity inside a tumour. A limitation that applies to all imaging modalities is that the image interpretation is based on a visual process. However, there are features within each image that may not be appreciated readily by the naked eye [3].

Recently, there has been extensive effort in the medical imaging community to obtain correlations between imaging features and tumour heterogeneity; an approximation for this concern is radiomics, which is a new technique that depends on the extraction of more data from biomedical images that cannot be investigated by the naked eye. This term was used for first time by Lambin et al., who investigated quantitative image analysis such as texture analysis to assess tumour heterogeneity [4]. Several studies demonstrated a promising role for radiomics in cancer diagnosis, staging, and treatment assessment $[5,6]$.

No standardisation has yet been developed for radiomics. Radiomics features are variable through many factors such as scanners, test-retest, observers, segmentation methods, and image reconstruction [7-9]. In addition, the mode of imaging may have an influence on radiomics. Texture analysis was performed in $2 \mathrm{D}$ mode. However, the advancements in 3D information acquisition and the high spatial resolution allow better capture of tissue properties [10]. Few studies have looked at the differences between radiomics or textural features in 3D and 2D modes utilising MRI or CT [11,12]. Ortiz-Ramón et al. introduced a radiomics approach on MRI of cancer lesions including lung cancer and melanoma [13].

The aim of our work was to evaluate the correlation of $2 \mathrm{D}$ and $3 \mathrm{D}$ radiomics and metabolic features of hepatocellular carcinoma with tumour diameter, staging, and metabolic tumour volume.

\section{Material and methods}

\section{Patients}

This retrospective study included 33 consecutive patients with HCC proven by histopathology ( 29 males and $4 \mathrm{fe}$ males) between November 2016 and April 2018. Patients were referred to our department primarily to investigate the extra-hepatic disease before starting an appropriate management plan. The average age was 57.7 years (range 39-77). Disease stage was assigned by a tumour board committee and based on the American Joint Committee on Cancer (AJCC) cancer staging manual 2017 [14].
The primary HCC was diagnosed by histopathology, the nodal and metastatic disease was established with reference to dedicated imaging, histopathology/histocytology of suspicious lesions when indicated to change line of management as well as the clinical assessment and follow-up. After staging, patients were referred to a multidisciplinary HCC panel including an oncologist, surgeon, hepatologist, and interventional radiologist. The management plan was implemented based on the Barcelona Clinic Liver Cancer (BCLC) system and its updates, which incorporates tumour morphology, liver function, and health performance status along with treatmentdependent variables $[15,16]$.

The study was approved by the institutional review board (IRB), and informed consent was waved.

\section{Imaging techniques}

The study was performed using a PET/CT scanner (Siemens Biograph 128-mCT). The patients were positioned in the PET/CT scanner approximately after injection of FDG intravenously the patients injected according to their weight by $0.1 \mathrm{mCi}$ or $3.7 \mathrm{MBq}$ for each $\mathrm{kg}$. A non-contrast CT scan was acquired from the base of the skull to the upper high region and used for attenuation correction. Images size was $200 \times 200$ pixels; slice thickness $=1 \mathrm{~mm}$.

\section{Image processing and analysis}

Imaging interpretation and analysis were performed and revised by a qualified radiologist with 15 years' experience in reading $\mathrm{PET} / \mathrm{CT}$. The tumours were segmented from the PET images after CT correction, using a semiautomatic method by ITK-snap software version 3.6.0 in 2D mode. In 3D mode the Pet scan segmented directly using a semiautomatic method in LIFEx package version 4.0.0 (https://www.lifexsoft.org/) [17]. The metabolic parameters and texture features were extracted using the LIFEx package. Figure 1 shows a diagram of the workflow of radiomics extraction, and Figure 2 shows a PET scan before and after delineation in the LIFEx package.

\section{Metabolic parameters}

The standardised uptake value (SUV) is defined as the tissue concentration of tracer, as measured by a PET scanner, divided by the activity injected divided by body weight [18]. There are many parameters related to SUV that can be useful for cancer diagnosis and staging.

SUV $_{\text {max }}$ : The maximum SUV value at the region of interest, which is the commonly used SUV clinically; however, some works show that another SUV factor can give a global view for tumours, such as metabolic tumour volume (MTV) and TLG.

SUV $_{\text {mean }}$ : The average SUV value at the region of interest. 


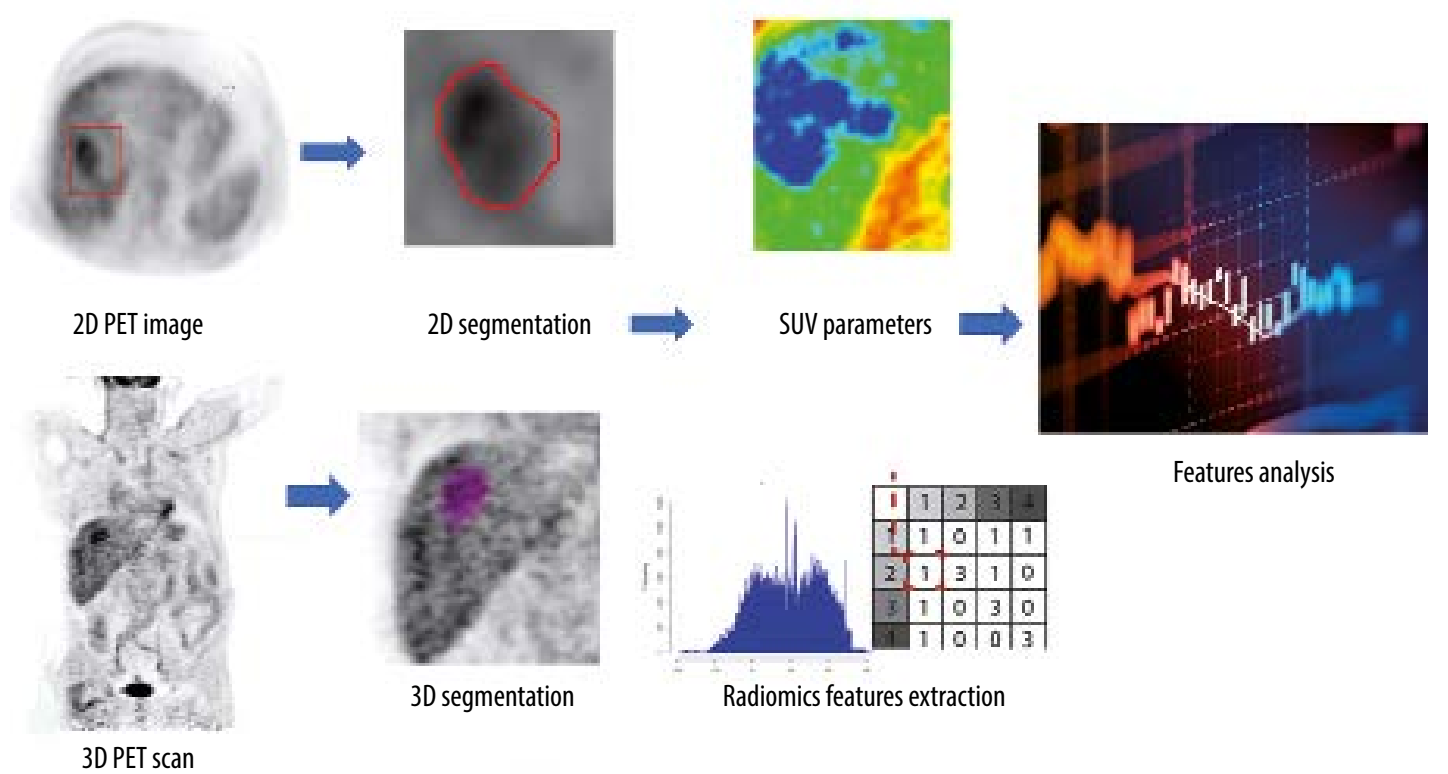

Figure 1. The workflow of standardised uptake value (SUV) parameters and radiomics features extraction
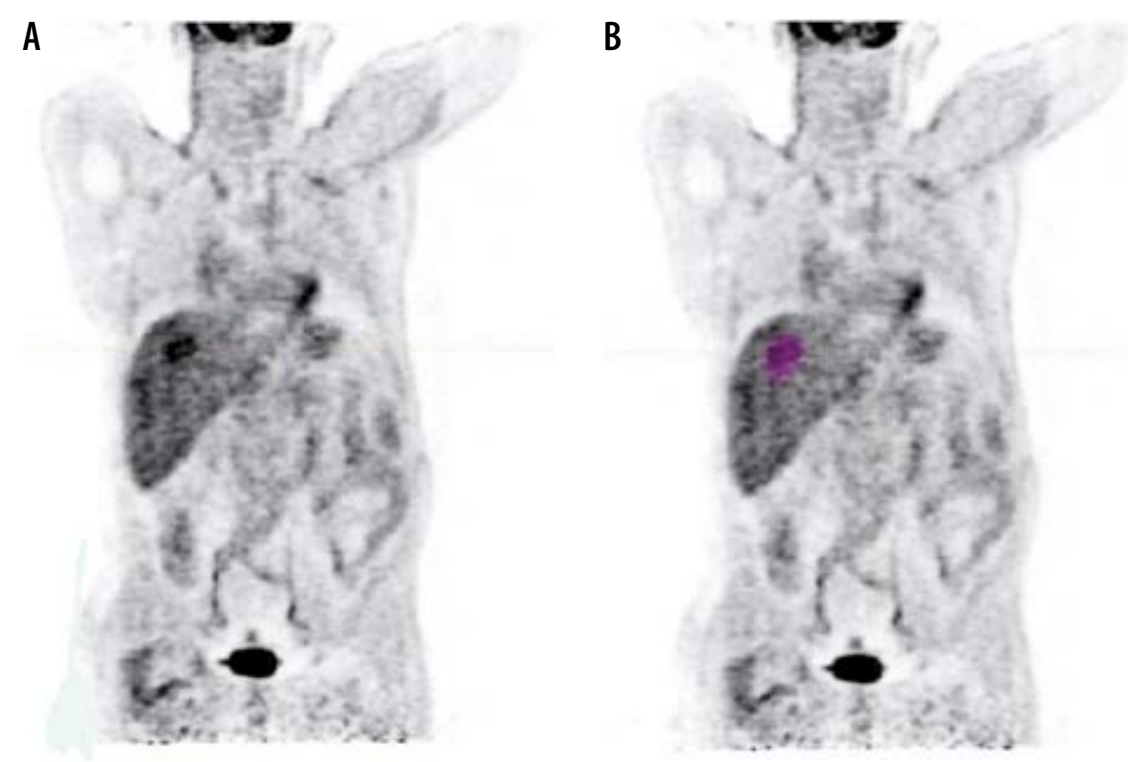

Figure 2. Coronal positron-emission tomography (PET) images of a 59-year-old male with hepatocellular carcinoma before (A) and after (B) delineation of the liver lesion using LIFEx package terest.

$\mathrm{SUV}_{\min }$ : The minimum SUV value at the region of in-

Metabolic tumour volume (MTV): Measures the active volume in $\mathrm{ml}$.

Total lesion glycolysis (TLG): Is defined as the product of SUV ${ }_{\text {mean }}$ and MTV [19].

\section{Intra-tumour heterogeneity}

The ${ }^{18} \mathrm{~F}-\mathrm{FDG}$ uptake heterogeneity was estimated using the coefficient of variation (COV), defined as the ratio between the standard deviation of SUV values and the mean SUV value within the delineated MTV [20].

SUV parameters are referred to as usual metabolic parameters; MTV and TLG are referred to as global metabolic parameters [21].

\section{Texture features}

Thirty-five radiomics features are studied. Histogram indices derived after determination of bin width; the grey level co-occurrence matrix (GLCM) takes into consideration the arrangements of pairs of voxels to calculate textural indices, and the neighbourhood grey-level different matrix (NGLDM) corresponds to the distinction of grey-level between one voxel and its 26 neighbours in 3 dimensions. The grey-level run length matrix (GLRLM) provides the scale of consistent runs for every grey-level. The grey-level zone length matrix (GLZLM) provides data on the scale of consistent zones for every grey-level in 3 dimensions.

Table 1 summarises all of the different features included in this study. More details about the radiomics features used 
Table 1. Summary of the features included in this study

\begin{tabular}{|c|c|c|}
\hline Feature name & Feature index & Feature type \\
\hline The minimum SUV & $S_{U V}$ min $(S U V)$ & SUV/normal metabolic parameters \\
\hline The average SUV & $S_{U} V_{\text {mean }}(S U V)$ & SUV/normal metabolic parameters \\
\hline The standard deviation of SUV & SUV $_{\text {std }}($ SUV) & SUV/normal metabolic parameters \\
\hline The maximum SUV & SUV $_{\max }($ SUV) & SUV/normal metabolic parameters \\
\hline The coefficient of variation & SUV $_{\text {(std/mean) }}$ & SUV/normal metabolic parameters \\
\hline Total lesion glycolysis & TLG (ml) & Global metabolic parameters \\
\hline Metabolic tumour volume & (MTV) Volume (ml) & Global metabolic parameters \\
\hline Skewness & HIST0_Skewness & Histogram indices \\
\hline Kurtosis & HISTO_Kurtosis & Histogram indices \\
\hline Entropy & HISTO_Entropy_log10 & Histogram indices \\
\hline Entropy & HISTO_Entropy_log2 & Histogram indices \\
\hline Energy & HISTO_Energy & Histogram indices \\
\hline Homogeneity & GLCM_Homogeneity & The grey level co-occurrence matrix (GLCM) \\
\hline Energy & GLCM_Energy & GLCM \\
\hline Contrast & GLCM_Contrast & GLCM \\
\hline Correlation & GLCM_Correlation & GLCM \\
\hline Entropy & GLCM_Entropy_log10 & GLCM \\
\hline Entropy & GLCM_Entropy_log2 & GLCM \\
\hline Dissimilarity & GLCM_Dissimilarity & GLCM \\
\hline Short-run emphasis & GLRLM_SRE & The grey-level run length matrix (GLRLM) \\
\hline Long-run emphasis & GLRLM_LRE & GLRLM \\
\hline Low grey-level run emphasis & GLRLM_LGRE & GLRLM \\
\hline High grey-level run emphasis & GLRLM_HGRE & GLRLM \\
\hline Short-run low grey-level emphasis & GLRLM_SRLGE & GLRLM \\
\hline Short-run high grey-level emphasis & GLRLM_SRHGE & GLRLM \\
\hline Long-run low grey-level emphasis & GLRLM_LRLGE & GLRLM \\
\hline Long-run high grey-level emphasis & GLRLM_LRHGE & GLRLM \\
\hline Grey-level non-uniformity for run & GLRLM_GLNU & GLRLM \\
\hline Run length non-uniformity & GLRLM_RLNU & GLRLM \\
\hline Run percentage & GLRLM_RP & GLRLM \\
\hline Coarseness & NGLDM_Coarseness & The neighbourhood grey-level different matrix (NGLDM) \\
\hline Contrast & NGLDM_Contrast & NGLDM \\
\hline Busyness & NGLDM_Busyness & NGLDM \\
\hline Short-zone emphasis & GLZLM_SZE & The grey-level zone length matrix (GLZLM) \\
\hline Long-zone emphasis & GLZLM_LZE & GLZLM \\
\hline Low grey-level zone emphasis & GLZLM_LGZE & GLZLM \\
\hline High grey-level zone emphasis & GLZLM_HGZE & GLZLM \\
\hline Short-zone low grey-level emphasis & GLZLM_SZLGE & GLZLM \\
\hline Short-zone high grey-level emphasis & GLZLM_SZHGE & GLZLM \\
\hline Long-zone low grey-level emphasis & GLZLM_LZLGE & GLZLM \\
\hline Long-zone high grey-level emphasis & GLZLM_LZHGE & GLZLM \\
\hline Grey-level non-uniformity for zone & GLZLM_GLNU & GLZLM \\
\hline Zone length non-uniformity & GLZLM_ZLNU & GLZLM \\
\hline Zone percentage & GLZLM_ZP & GLZLM \\
\hline
\end{tabular}



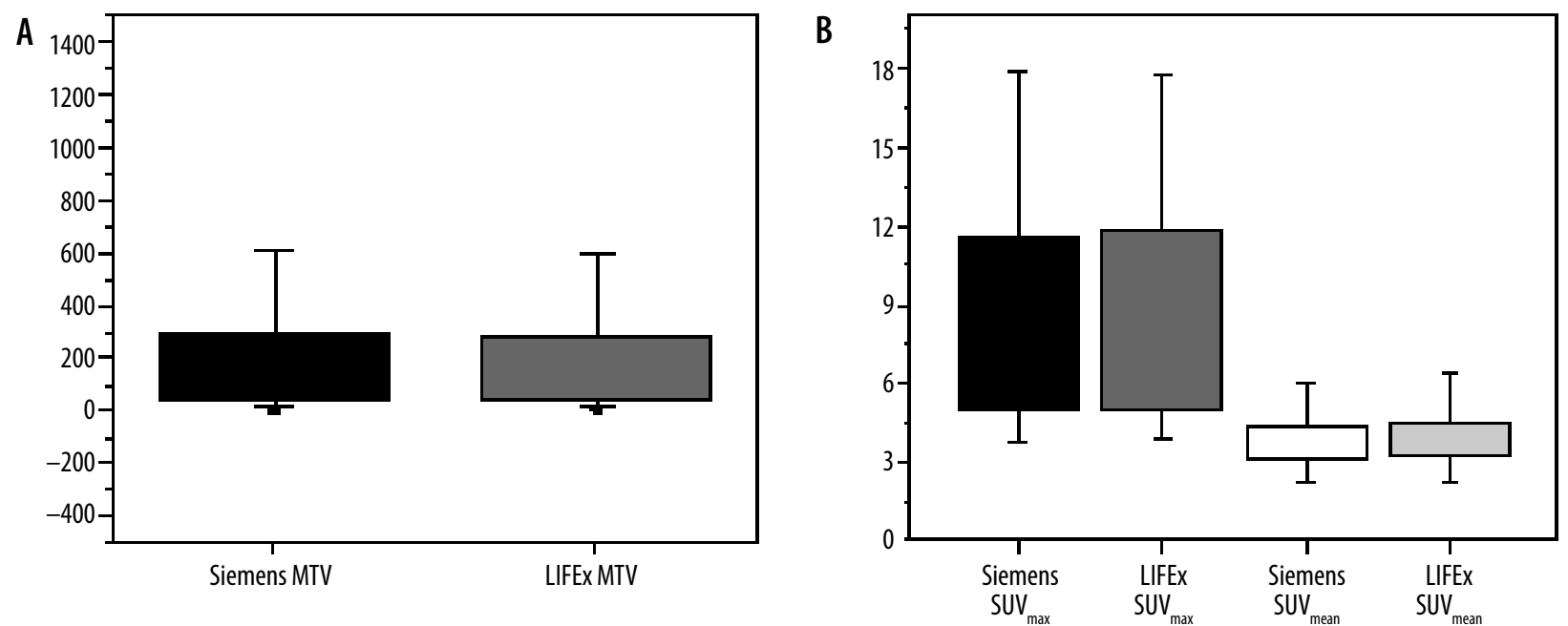

Figure 3. Show a box plot of metabolic parameters in Siemens vs. LIFEx software. A) Metabolic tumour volume (MTV) in both software. B) A SUV max $_{\text {and }}$ SUV $_{\text {mean }}$ in each software

in this study can be found at: https://lifexsoft.org/index. php/resources/19-texture/radiomicfeatures?filter_tag [0]=.

\section{Statistical analysis}

A paired $t$-test was used to obtain the differences between the metabolic and radiomics features in $3 \mathrm{D}$ and $2 \mathrm{D}$ mode, and then the Spearman correlation coefficient was calculated to study the relationship between metabolic and radiomics features and the tumour staging, diameter, and metabolic volume. All statistical tests were calculated using Origin lab software version 6 and IBM-SPSS version 19.

\section{Results}

SUV values ( $\mathrm{SUV}_{\text {max }}, \mathrm{SUV}_{\text {mean }}$, and MTV) were compared between the LIFEx package and an approved software: Siemens Syngo trueD. An independent $t$-test for the values in both software calculated to measure the differences between the values. SUV ${ }_{\text {mean }}$ was the most stable feature between the two software packages, with a significance level $(\rho)=0.91$, where MTV and $\mathrm{SUV}_{\max }$ gave $(\rho)=0.87$ and 0.61 , respectively. There was no significant difference between the two software packages, as shown in Figure 3.

The variations between SUV parameters, except $\mathrm{SUV}_{\text {min' }}$, and 20 radiomics features were statistically stable in $3 \mathrm{D}$ and $2 \mathrm{D}$ modes, as shown in Table 2. Most features had $\rho$-values higher than 0.05; the most stable in the SUV feature was $\mathrm{SUV}_{\text {mean }}$, with $\rho=0.588$, while in radiomics features the most stable were GLCM_Contrast and GLZLM_LGZE, with $\rho=0.89$ and 0.82 , respectively. Around 15 features were significantly different between the 2 modes; the most significant was GLZLM_ZP, with $\rho \leq 0.001$.

Spearman correlation coefficients for both $3 \mathrm{D}$ and $2 \mathrm{D}$ features with tumour maximum diameter, tumour staging, and tumour metabolic volume are shown in Tables 3 and 4, respectively. Most of 3D mode features showed high correlation with metabolic tumour volume, as shown in Table 3; the strongest correlation of metabolic parameters was found with TLG, with rs $=0.98$, and from radiomics features it was GLRLM_RLNU, with rs $=0.97$. Considering the $2 \mathrm{D}$ mode features, there was no significant correlation between the SUV as well as radiomics features and MTV, AJCC staging, or tumour maximum diameter, where all spearman correlation coefficients were less than 0.3 , as shown in Table 4.

To compare HCC tumour heterogeneity through uptake heterogeneity and radiomics features, the relationship between SUV (std/mean) and GLCM entropy with metabolic tumour volume is shown in Figure 4. The ${ }^{18} \mathrm{~F}-\mathrm{FDG}$ uptake heterogeneity coefficient $\mathrm{SUV}_{\text {(st/mean) }}$ mean value $=$ $0.304 \pm 0.14$, and GLCM-entropy radiomics feature mean $=2.016 \pm 0.523$.

\section{Discussion}

Several studies have demonstrated agreement on the usefulness of ${ }^{18} \mathrm{~F}$ [FDG]-PET/CT in defining HCC, staging, and treatment assessment; furthermore, some authors reported a high correlation with histopathology results [22-25]. Our study showed that the addition of radiomics features to PET images can provide more information about cancer structure and intratumour heterogeneity.

Comparing the SUV parameters generated by LIFEx, which is an open-source software, with Siemens Syngovia TrueD (commercial software), there was no significant difference between the two software programs. Arian et al. reported differences in SUV parameters through four platforms [26]. On the other hand, Kenny et al. compared SUV parameters through 14 software programs using three phantoms calibrated on 3 PET/CT scanners, and agreement found in some software included Siemens TrueD [27].

In the current work we found that $\mathrm{SUV}_{\text {mean }}$ and $\mathrm{SUV}_{\text {(std/mean) }}$ were smaller in $3 \mathrm{D}$ than in $2 \mathrm{D}$ mode, whereas, 
Table 2. The results of paired $t$-test for metabolic and radiomics features in both 2D and 3D modes; the cells signed with ${ }^{*}$ ) show the significant difference in features between $3 \mathrm{D}$ and $2 \mathrm{D}$

\begin{tabular}{|c|c|c|}
\hline Features & $t$ & $p$-value \\
\hline SUV $_{\text {(std/mean) }}$ & 1.377 & 0.1782 \\
\hline SUV $_{\min }$ (SUV) & -5.557 & $<0.0001^{*}$ \\
\hline$S U V_{\text {mean }}(S U V)$ & -0.546 & 0.588 \\
\hline SUV $_{\text {std }}$ (SUV) & 0.767 & 0.4484 \\
\hline SUV $_{\max }($ SUV) & 1.278 & 0.2104 \\
\hline HISTO_Skewness & 0.550 & 0.5858 \\
\hline HISTO_Kurtosis & 0.697 & 0.4908 \\
\hline HISTO_Entropy_log10 & 1.491 & 0.1458 \\
\hline HISTO_Entropy_log2 & 1.491 & 0.1458 \\
\hline HISTO_Energy & 1.955 & 0.0594 \\
\hline GLCM_Homogeneity & 0.754 & 0.4562 \\
\hline GLCM_Energy & -1.536 & 0.1345 \\
\hline GLCM_Contrast & -0.144 & 0.8861 \\
\hline GLCM_Correlation & 3.594 & $0.0011^{*}$ \\
\hline GLCM_Entropy_log10 & 2.489 & $0.0182^{*}$ \\
\hline GLCM_Entropy_log2 & 2.489 & $0.0182^{*}$ \\
\hline GLCM_Dissimilarity & -0.277 & 0.7833 \\
\hline GLRLM_SRE & -1.099 & 0.2800 \\
\hline GLRLM_LRE & 0.475 & 0.6377 \\
\hline GLRLM_LGRE & -0.742 & 0.4633 \\
\hline GLRLM_HGRE & -0.28 & 0.7816 \\
\hline GLRLM_SRLGE & -0.575 & 0.5691 \\
\hline GLRLM_SRHGE & -0.338 & 0.7372 \\
\hline GLRLM_LRLGE & -0.952 & 0.3485 \\
\hline GLRLM_LRHGE & 0.586 & 0.5620 \\
\hline GLRLM_GLNU & 5.845 & $<0.0001^{*}$ \\
\hline GLRLM_RLNU & 4.837 & $<0.0001^{*}$ \\
\hline GLRLM_RP & -0.681 & 0.5007 \\
\hline NGLDM_Coarseness & -10.763 & $<0.001$ \\
\hline NGLDM_Contrast & -2.763 & $0.0094^{*}$ \\
\hline NGLDM_Busyness & 4.705 & $<0.0001^{*}$ \\
\hline GLZLM_SZE & -11.597 & $<0.0001^{*}$ \\
\hline GLZLM_LZE & 3.020 & $0.0049^{*}$ \\
\hline GLZLM_LGZE & -0.225 & 0.8234 \\
\hline GLZLM_HGZE & 0.401 & 0.6908 \\
\hline GLZLM_SZLGE & -1.928 & 0.0628 \\
\hline GLZLM_SZHGE & -1.526 & 0.0628 \\
\hline GLZLM_LZLGE & 2.243 & $0.0319^{*}$ \\
\hline GLZLM_LZHGE & 3.089 & $0.0041^{*}$ \\
\hline GLZLM_GLNU & 4.430 & $0.0001^{*}$ \\
\hline GLZLM_ZLNU & 2.586 & $0.0145^{*}$ \\
\hline GLZLM_ZP & -12.754 & $<0.0001^{*}$ \\
\hline
\end{tabular}

$\mathrm{SUV}_{\max }$ was little higher in 2D mode than in $3 \mathrm{D}$ mode. Kocabaş et al. reported that $\mathrm{SUV}_{\max }$ was variable between $3 \mathrm{D}$ and $2 \mathrm{D}$ modes and the values were smaller in $3 \mathrm{D}$ mode [28]. In the current work we found that $\mathrm{SUV}_{\text {mean }}$ and $\mathrm{SUV}_{\text {(std/mean) }}$ were smaller in $3 \mathrm{D}$ than in $2 \mathrm{D}$ mode, whereas $\mathrm{SUV}_{\max }$ was a little larger in 2D mode than in 3D mode.

Our study showed a strong correlation of 3D mode features, especially TLG and GLRLM_RLNU with MTV. This strong correlation could be explained by the fact that TLG and GLRLM_RLNU voxel values are not absolute SUV values and depend on the lesion volume. Therefore, it showed a strong correlation with MTV. The lowest correlation of MTV was found with GLRLM_LRLGE. A similar correlation between the same feature and MTV was found by Vicente et al. in the case of breast cancer dual time acquisition PET [29]. GLRLM_LRLGE measures the roughness of the images, which increases when the texture is dominated by long runs that have low grey levels; hence, it may not correlate with volume.

In this study, TLG from metabolic parameters and GLRLM_RLNU from radiomics features demonstrated a strong correlation with maximum diameter of the lesion, with rs $=0.75$ and 0.71 , respectively. Hatt et al. reported that MTV has a close correlation with tumour diameter in lung cancer [20]. So, the same correlation can be found in the case of TLG because it depends on MTV, and as we mentioned before: RLNU is correlated to MTV and to the diameter. Regarding AJCC staging, some features showed a medium correlation with metabolic and radiomics features; the strongest was GLZLM_GLNU, with $\mathrm{rs}=0.4915$. Van Go et al. reported a similar correlation between some radiomics features of $\left[{ }^{18} \mathrm{~F}\right]$ (FDG-PET) images and AJCC staging for non-small cell lung carcinoma (NSCLC) [21].

Considering the $2 \mathrm{D}$ mode features, there was no significant correlation between the SUV as well as radiomics features and MTV, AJCC staging, or tumour maximum diameter. This may be because the spatial resolution of PET images is low, which does not give strong texture information in small areas [18].

It has been shown that tumour heterogeneity increases with larger tumour volume, as reported by Hatt et al. and Brooks and Grigsby [20,30]. In our cohort we found that some HCC tumours may be more heterogeneous in both uptake and radiomics value without being large in volume, as shown in Figure 5. SUV ${ }_{\max }$ is the commonest parameter used for follow-up; however, it suffers from the fact that it is not correlated to tumour volume and tumour heterogeneity. Figure 5 demonstrates a tumour with high $\mathrm{SUV}_{\max }$, but its textural analysis is less heterogeneous than another tumour with lower $\mathrm{SUV}_{\max }$ value. Hatt et al. suggested that GLCM entropy with MTV is lower in the volume range less than $50 \mathrm{~cm}^{3}$ [31]; this finding is in agreement with our study.

Limitations of our study include the relatively small sample size, restricted number of radiomics features, and 
Table 3. Spearman correlation coefficient rs and $\rho$-values for metabolic and radiomics features in 3D mode with tumour metabolic volume, tumour staging, and maximum diameter; cells signed with $\left(^{*}\right)$ are medium correlation where cells signed with $\left(^{* *}\right)$ are high correlation

\begin{tabular}{|c|c|c|c|c|c|c|}
\hline 3D features & Diameter (rs) & Diameter $(\rho)$ & $\begin{array}{l}\text { AJCC staging } \\
\text { (rs) }\end{array}$ & $\begin{array}{l}\text { AJCC staging } \\
(\rho)\end{array}$ & MTV (rs) & MTV (p) \\
\hline$S U V_{\text {meanstd }}$ & 0.5019 & $0.0029^{* *}$ & 0.3242 & $0.0657^{*}$ & 0.6090 & $0.0002^{* *}$ \\
\hline$S_{\text {SUV }}$ (SUV) & -0.5439 & $0.0011^{* *}$ & -0.2844 & 0.1087 & -0.5505 & $0.0009^{* *}$ \\
\hline$S_{\text {SUV }}$ (SUV) & 0.3246 & $0.0654^{*}$ & 0.4177 & $0.0156^{*}$ & 0.5114 & $0.0024^{* *}$ \\
\hline SUV Std $_{\text {(SUV) }}$ & 0.4400 & $0.0104^{*}$ & 0.3509 & $0.0453^{*}$ & 0.6123 & $0.0002^{* *}$ \\
\hline SUV $_{\max }$ (SUV) & 0.4882 & $0.0040^{*}$ & 0.4108 & $0.0176^{*}$ & 0.6928 & $<0.0001^{* *}$ \\
\hline SUV $_{\text {peak }} 1 \mathrm{ml}$ & 0.4903 & 0.0038 & 0.3799 & $0.0292^{*}$ & 0.6801 & $<0.0001^{* *}$ \\
\hline $\mathrm{TLG}(\mathrm{ml})$ & 0.7461 & $<0.0001^{* *}$ & 0.4556 & $0.0077^{*}$ & 0.9776 & $<0.0001^{* *}$ \\
\hline HISTO_Skewness & 0.3281 & $0.0623^{*}$ & 0.1334 & 0.4592 & 0.4679 & $0.006^{*}$ \\
\hline HISTO_Kurtosis & 0.2966 & 0.0937 & -0.0407 & 0.8222 & 0.3382 & $0.0542^{*}$ \\
\hline HISTO_Entropy_log10 & 0.4500 & $0.0086^{*}$ & 0.3554 & $0.0424^{*}$ & 0.6230 & $0.0001^{* *}$ \\
\hline HISTO_Energy & -0.4323 & $0.0120^{*}$ & -0.3452 & $0.0491^{*}$ & -0.5946 & $0.0003^{* *}$ \\
\hline Sphericity (only for 3D ROI) & -0.2573 & 0.1483 & -0.4117 & $0.0173^{*}$ & -0.5003 & $0.0030^{* *}$ \\
\hline SHAPE_Compacity ROI (nZ > 1) & 0.7363 & $<0.0001^{* *}$ & 0.3128 & $0.0764^{*}$ & 0.9542 & $<0.0001^{* *}$ \\
\hline GLCM_Homogeneity & -0.2991 & 0.0908 & -0.3118 & $0.0773^{*}$ & -0.4602 & $0.007^{*}$ \\
\hline GLCM_Energy & -0.4090 & $0.0181^{*}$ & -0.3576 & $0.041^{*}$ & -0.5872 & $0.0003^{* *}$ \\
\hline GLCM_Contrast & 0.3269 & $0.0633^{*}$ & 0.3358 & $0.0561^{*}$ & 0.4773 & $0.0050^{*}$ \\
\hline GLCM_Correlation & 0.6277 & $<0.0001^{* *}$ & 0.2483 & 0.1635 & 0.7614 & $<0.0001^{* *}$ \\
\hline GLCM_Entropy_log10 & 0.4323 & $0.0120^{*}$ & 0.3805 & $0.0289^{*}$ & 0.6096 & $0.0002^{* *}$ \\
\hline GLCM_Dissimilarity & 0.3145 & $0.0746^{*}$ & 0.3340 & $0.0575^{*}$ & 0.4766 & $0.0050^{*}$ \\
\hline GLRLM_SRE & 0.1169 & 0.5190 & 0.2701 & 0.1285 & 0.2012 & 0.2615 \\
\hline GLRLM_LRE & -0.0875 & 0.6283 & -0.2556 & 0.1511 & -0.1310 & 0.4674 \\
\hline GLRLM_LGRE & -0.1273 & 0.4802 & -0.2668 & 0.1333 & -0.2707 & 0.1276 \\
\hline GLRLM_HGRE & 0.3460 & $0.0486^{*}$ & 0.4115 & $0.0174^{*}$ & 0.5394 & $0.0012^{* *}$ \\
\hline GLRLM_SRLGE & -0.1521 & 0.3982 & -0.324 & $0.0658^{*}$ & -0.3185 & $0.0708^{*}$ \\
\hline GLRLM_SRHGE & 0.3314 & $0.0596^{*}$ & 0.4218 & $0.0145^{*}$ & 0.5124 & $0.0023^{* *}$ \\
\hline GLRLM_LRLGE & -0.0087 & 0.9617 & -0.1133 & 0.5303 & -0.0745 & 0.6802 \\
\hline GLRLM_LRHGE & 0.3883 & $0.0256^{*}$ & 0.4538 & $0.0080^{*}$ & 0.6380 & $<0.0001^{* *}$ \\
\hline GLRLM_GLNU & 0.6943 & $<0.0001^{* *}$ & 0.2672 & 0.1328 & 0.8930 & $<0.0001^{* *}$ \\
\hline GLRLM_RLNU & 0.7143 & $<0.0001^{* *}$ & 0.4324 & $0.0120^{*}$ & 0.9733 & $<0.0001^{* *}$ \\
\hline GLRLM_RP & 0.1074 & 0.5519 & 0.2334 & 0.1911 & 0.1852 & 0.3023 \\
\hline NGLDM_Coarseness & -0.711 & $<0.0001^{* *}$ & -0.3993 & $0.0213^{*}$ & -0.9652 & $<0.0001^{* *}$ \\
\hline NGLDM_Contrast & -0.0054 & 0.9764 & 0.181 & 0.3135 & 0.0896 & 0.6201 \\
\hline NGLDM_Busyness & 0.3197 & $0.0697^{*}$ & 0.0937 & 0.6041 & 0.4676 & $0.0061^{*}$ \\
\hline GLZLM_SZE & 0.3018 & $0.0878^{*}$ & 0.46 & $0.0071^{*}$ & 0.3061 & $0.0831^{*}$ \\
\hline GLZLM_LZE & 0.1057 & 0.5581 & -0.1296 & 0.4722 & 0.1407 & 0.4348 \\
\hline GLZLM_LGZE & -0.311 & $0.0781^{*}$ & -0.4422 & $0.0100^{*}$ & -0.5043 & $0.0028^{* *}$ \\
\hline GLZLM_HGZE & 0.4122 & $0.0171^{*}$ & 0.4149 & $0.0163^{*}$ & 0.6243 & $0.0001^{* *}$ \\
\hline GLZLM_SZLGE & -0.3756 & $0.0312^{*}$ & -0.3683 & $0.0350^{*}$ & -0.6233 & $0.0001^{* *}$ \\
\hline GLZLM_SZHGE & 0.4042 & $0.0197^{*}$ & 0.4110 & $0.0175^{*}$ & 0.5929 & $0.0003^{* *}$ \\
\hline GLZLM_LZLGE & 0.1910 & 0.2869 & -0.0546 & 0.7627 & 0.2176 & 0.2239 \\
\hline GLZLM_LZHGE & 0.1793 & 0.3180 & -0.1036 & 0.5660 & 0.2654 & 0.1355 \\
\hline GLZLM_GLNU & 0.6590 & $<0.0001^{* *}$ & 0.4915 & $0.0037^{*}$ & 0.9181 & $<0.0001^{* *}$ \\
\hline GLZLM_ZLNU & 0.6412 & $<0.0001^{* *}$ & 0.4607 & $0.0070^{*}$ & 0.8590 & $<0.0001^{* *}$ \\
\hline GLZLM_ZP & 0.1273 & 0.4802 & 0.2988 & 0.0912 & 0.1888 & 0.2926 \\
\hline
\end{tabular}


Table 4. Spearman correlation coefficient (rs) and p-values for metabolic and radiomics features in 2D mode with tumour metabolic volume, tumour staging, and maximum diameter

\begin{tabular}{|c|c|c|c|c|c|c|}
\hline 2D features & $\begin{array}{c}\text { Diameter } \\
\text { (rs) }\end{array}$ & $\begin{array}{c}\text { Diameter } \\
(p)\end{array}$ & $\begin{array}{l}\text { AJCC staging } \\
\text { (rs) }\end{array}$ & $\begin{array}{c}\text { AJCC satging } \\
(p)\end{array}$ & $\begin{array}{l}\text { MTV } \\
\text { (rs) }\end{array}$ & $\begin{array}{l}\text { MTV } \\
(p)\end{array}$ \\
\hline SUV $V_{\text {mean/std }}$ & -0.0368 & 0.8389 & 0.0310 & 0.8638 & -0.0842 & 0.6412 \\
\hline SUV $_{\min }(\mathrm{SUV})$ & 0.1086 & 0.5475 & -0.0033 & 0.9856 & 0.1889 & 0.2925 \\
\hline$S U V_{\text {mean }}(S U V)$ & 0.0746 & 0.6798 & 0.0969 & 0.5915 & 0.1300 & 0.4708 \\
\hline SUV $_{\text {std }}$ (SUV) & -0.0666 & 0.7127 & 0.0209 & 0.9082 & -0.0154 & 0.9323 \\
\hline SUV $_{\max }($ SUV) & 0.0698 & 0.6996 & 0.0830 & 0.6400 & 0.1183 & 0.5119 \\
\hline HISTO_Skewness & 0.0740 & 0.6825 & 0.0924 & 0.6090 & 0.0568 & 0.7535 \\
\hline HISTO_Kurtosis & 0.1302 & 0.4703 & -0.1278 & 0.4785 & 0.0739 & 0.6829 \\
\hline HISTO_Entropy_log10 & -0.0746 & 0.6798 & 0.0162 & 0.9289 & -0.0294 & 0.8709 \\
\hline HISTO_Energy & 0.0289 & 0.8730 & -0.0172 & 0.9241 & 0.0137 & 0.9397 \\
\hline GLCM_Homogeneity & 0.0612 & 0.7350 & -0.0419 & 0.8168 & 0.0160 & 0.9294 \\
\hline GLCM_Energy & 0.0256 & 0.8876 & -0.0064 & 0.9720 & 0.0160 & 0.9294 \\
\hline GLCM_Contrast & -0.1024 & 0.5707 & 0.0592 & 0.7436 & -0.0475 & 0.7931 \\
\hline GLCM_Correlation & 0.0492 & 0.7857 & -0.0007 & 0.9968 & 0.0150 & 0.9338 \\
\hline GLCM_Entropy_log10 & -0.0385 & 0.8316 & -0.0167 & 0.9265 & -0.0053 & 0.9764 \\
\hline GLCM_Dissimilarity & -0.0920 & 0.6105 & 0.0494 & 0.7849 & -0.0331 & 0.8549 \\
\hline GLRLM_SRE & -0.0669 & 0.7114 & -0.0719 & 0.6910 & -0.0104 & 0.9544 \\
\hline GLRLM_LRE & 0.1066 & 0.5550 & -0.0719 & 0.6910 & 0.0468 & 0.7959 \\
\hline GLRLM_LGRE & -0.1384 & 0.4425 & -0.077 & 0.6703 & -0.1842 & 0.3049 \\
\hline GLRLM_HGRE & 0.0815 & 0.6522 & 0.0782 & 0.6650 & 0.1257 & 0.4859 \\
\hline GLRLM_SRLGE & -0.1663 & 0.3550 & -0.0824 & 0.6484 & -0.2276 & 0.2027 \\
\hline GLRLM_SRHGE & 0.0882 & 0.6256 & 0.0868 & 0.6311 & 0.1270 & 0.4812 \\
\hline GLRLM_LRLGE & -0.0728 & 0.6873 & -0.0866 & 0.6320 & -0.1217 & 0.5000 \\
\hline GLRLM_LRHGE & 0.1755 & 0.3286 & 0.0902 & 0.6180 & 0.2704 & 0.1280 \\
\hline GLRLM_GLNU & 0.1494 & 0.4066 & -0.2200 & 0.2180 & 0.0956 & 0.5967 \\
\hline GLRLM_RLNU & 0.1389 & 0.4409 & -0.0381 & 0.8330 & 0.0929 & 0.6070 \\
\hline GLRLM_RP & -0.0793 & 0.6609 & 0.0713 & 0.6932 & -0.0237 & 0.8957 \\
\hline NGLDM_Coarseness & -0.2543 & 0.1532 & -0.0378 & 0.8347 & -0.2129 & 0.2342 \\
\hline NGLDM_Contrast & -0.1594 & 0.3754 & 0.0084 & 0.9632 & -0.1080 & 0.5498 \\
\hline NGLDM_Busyness & 0.1300 & 0.4709 & 0.0336 & 0.8528 & 0.1003 & 0.5787 \\
\hline GLZLM_SZE & -0.0612 & 0.7350 & 0.0080 & 0.9640 & -0.0642 & 0.7227 \\
\hline GLZLM_LZE & 0.0766 & 0.6717 & -0.0944 & 0.6013 & 0.0254 & 0.8884 \\
\hline GLZLM_LGZE & -0.1466 & 0.4157 & -0.0920 & 0.6105 & -0.1999 & 0.2647 \\
\hline GLZLM_HGZE & 0.0863 & 0.6329 & 0.1106 & 0.5402 & 0.1337 & 0.4582 \\
\hline GLZLM_SZLGE & -0.1464 & 0.4163 & -0.0871 & 0.6290 & -0.2086 & 0.2441 \\
\hline GLZLM_SZHGE & 0.0447 & 0.8050 & 0.0986 & 0.5852 & 0.0889 & 0.6230 \\
\hline GLZLM_LZLGE & 0 & 1 & -0.0958 & 0.5957 & -0.0428 & 0.8131 \\
\hline GLZLM_LZHGE & 0.2553 & 0.1516 & 0.0425 & 0.8144 & 0.2707 & 0.1275 \\
\hline GLZLM_GLNU & 0.1516 & 0.3525 & -0.1690 & 0.3471 & 0.0789 & 0.6626 \\
\hline GLZLM_ZLNU & 0.0617 & 0.7329 & -0.0134 & 0.9409 & 0.0328 & 0.8564 \\
\hline GLZLM_ZP & -0.0795 & 0.6602 & 0.0801 & 0.6579 & -0.0428 & 0.8131 \\
\hline
\end{tabular}


lack of comparison of the findings with other clinical information such histopathology results. The clinical course of the disease and liver functions have not been compared with radiomics. However, the main focus of this investigation was to improve the performance of FDG-PET/CT in the diagnosis and characterisation of HCC, which will be extended in our future works to incorporate radiomics in clinical practice. Furthermore, there was medical value to our finding: we discovered the best mode for HCC radiomics for use in our future studies.

\section{Conclusions}

The metabolic parameters and radiomics features are variable between 3D and 2D modes. Some SUV parameters and radiomics features are statically stable through $3 \mathrm{D}$ and $2 \mathrm{D}$ modes. Image analysis in $3 \mathrm{D}$ radiomics features is significantly correlated with tumour volume, maximum diameter, and staging, whereas all features in $2 \mathrm{D}$ exhibit no correlation. Therefore, in comprehensive studies of intra-tumoral structure, 3D mode features can accurately evaluate tumour heterogeneity.
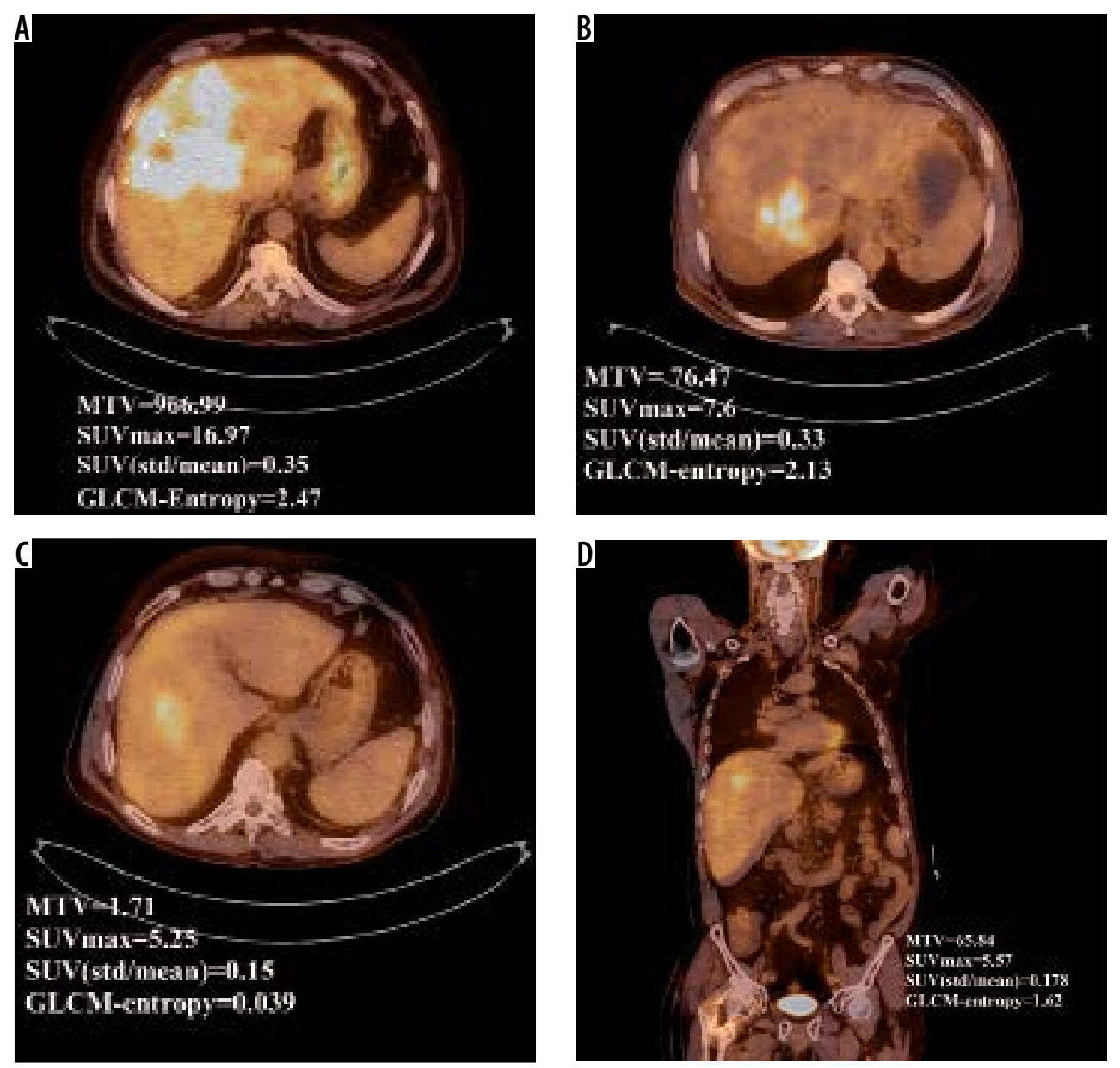

Figure 5. Comparison between two hepatocellular carcinoma (HCC) tumour parameters. (A) and (B) compare uptake heterogeneity coefficient and GLCMentropy of two HCC tumours. Although (B) have a higher metabolic tumour volume, it is more homogenous than tumour (A). (C) and (D) compare the parameters for the same patient in $2 \mathrm{D}$ and $3 \mathrm{D}$, respectively 
References

1. Balogh J, Iii Dv, Asham Eh, et al. Hepatocellular carcinoma : a review. J Hepatocell Carcinoma 2016; 3: 41-53.

2. Hammoud GM, Ibdah JA. Are we getting closer to understanding intratumor heterogeneity in hepatocellular carcinoma? Hepatobiliary Surg Nutr 2016; 5: 188-190.

3. Davnall F, Yip CSP, Ljungqvist G, et al. Assessment of tumor heterogeneity: an emerging imaging tool for clinical practice? Insights Imaging 2012; 3: 573-589.

4. Lambin P, Rios-velazquez E, Leijenaar R. Radiomics: extracting more information from medical images using advanced feature analysis. Eur J Cancer 2012; 48: 441-446.

5. Aerts HJWL, Velazquez ER, Leijenaar RTH, et al. Decoding tumour phenotype by noninvasive imaging using a quantitative radiomics approach. Nat Commun 2014; 5: 4006.

6. Coroller TP, Sc M, Grossmann P, et al. CT-based radiomic signature predicts distant metastasis in lung adenocarcinoma. Radiother Oncol 2016; 114: 345-350.

7. Mackin D, Fave X, Zhang L, et al. Measuring CT scanner variability of radiomics features. Invest Radiol 2015; 50: 757-765.

8. Parmar C, Leijenaar RTH, Grossmann P, et al. Radiomic feature clusters and prognostic signatures specific for lung and head and neck cancer. Sci Rep 2015; 5: 11044.

9. Altazi BA, Zhang GG, Fernandez DC, et al. Reproducibility of F18-FDG PET radiomic features for different cervical tumor segmentation methods, gray-level discretization, and reconstruction algorithms. J Appl Clin Med Phys 2017; 18: 32-48.

10. Depeursinge A, Foncubierta-Rodriguez A, Van De Ville D, Müller H. Three-dimensional solid texture analysis in biomedical imaging: review and opportunities. Medical Image Analysis 2014; 18: 176-196.

11. Mahmoud-Ghoneim D, Toussaint G, Constans JM, De Certaines JD Three dimensional texture analysis in MRI: A preliminary evaluation in gliomas. Magn Reson Imaging 2003; 21: 983-987.

12. Lubner MG, Stabo N, Lubner SJ, et al. CT textural analysis of hepatic metastatic colorectal cancer: pre-treatment tumor heterogeneity correlates with pathology and clinical outcomes. Abdom Imaging 2015; 40: 2331-2337.

13. Ortiz-Ramon R, Larroza A, Arana E, Moratal D. A radiomics evaluation of 2D and 3D MRI texture features to classify brain metastases from lung cancer and melanoma. Proc Annu Int Conf IEEE Eng Med Biol Soc 2017; 2017: 493-496.

14. Amin M, Edge S, Greene F, et al. (eds.). AJCC Cancer Staging Manual. Springer International Publishing 2017; 287-293.

15. Forner A, Reig M, Bruix J. Hepatocellular carcinoma. Lancet 2018; 391: 1301-1314.

16. Llovet JM, Brú C, Bruix J. Prognosis of hepatocellular carcinoma: the BCLC staging classification. Semin Liver Dis 1999; 19: 329-337.

17. Nioche $\mathrm{C}$, Orlhac F, Boughdad S, et al. Lifex: a freeware for radiomic feature calculation in multimodality imaging to accelerate advances in the characterization of tumor heterogeneity. Cancer Res 2018; 78: 4786-4789.
18. Keyes JW. SUV: standard uptake or silly useless value? J Nucl Med 1995; 36: 1836-1839.

19. Larson SM, Erdi Y, Akhurst T, et al. Tumor treatment response based on visual and quantitative changes in global tumor glycolysis using PET-FDG imaging. The visual response score and the change in total lesion glycolysis. Clin Positron Imaging 1999; 2: 159-171.

20. Hatt M, Cheze-le Rest C, van Baardwijk A, et al. Impact of tumor size and tracer uptake heterogeneity in 18F-FDG PET and CT non-small cell lung cancer tumor delineation. J Nucl Med 2011; 52: 1690-1697.

21. Van Gómez López O, García Vicente AM, Honguero Martínez AF, et al. Heterogeneity in [18 F]fluorodeoxyglucose positron emission tomography/computed tomography of non - small cell lung carcinoma and its relationship to metabolic parameters and pathologic staging. Mol Imaging 2014; 13; doi: 10.2310/7290.2014.00032.

22. Torizuka T, Tamaki N, Inokuma T, et al. In vivo assessment of glucose metabolism in hepatocellular carcinoma with FDG-PET. J Nucl Med 1995; 36: 1811-1817.

23. Wolfort RM, Papillion PW, Turnage RH, et al. Role of FDG-PET in the evaluation and staging of hepatocellular carcinoma with comparison of tumor size, AFP level, and histologic grade. Int Surg 2010; 95: 67-75.

24. Kitamura K, Hatano E, Higashi T, et al. Preoperative FDG-PET predicts recurrence patterns in hepatocellular carcinoma. Ann Surg Oncol 2012; 19: 156-162.

25. Dong A, Yu H, Wang Y, et al. FDG PET/CT and enhanced CT imaging of tumor heterogeneity in hepatocellular carcinoma: imaging-pathologic correlation. Clin Nucl Med 2014; 39: 808-810.

26. Arain Z, Lodge M, Wahl R. A comparison of SUV parameters across four commercial software platforms. J Nucl Med 2015; 56 (Suppl 3): 580.

27. Kenny B. SUV reproducibility on different reporting platforms What is SUV? BNMS spring meeting, Birmingham 2016.

28. Kocabas B, Yapar AF, Reyhan M, et al. Comparison of standardized uptake values obtained from $18 \mathrm{~F}$ fluorodeoxyglucose positron emission tomography/computed tomography imaging performed with $2 \mathrm{~d}$ and $3 \mathrm{D}$ modes in oncological cases. Diagnostic Interv Radiol 2012; 126-129.

29. Garcia-Vicente AM, Molina D, Pérez-Beteta J, et al. Textural features and SUV-based variables assessed by dual time point 18F-FDG PET/CT in locally advanced breast cancer. Ann Nucl Med 2017; 31: 726-735.

30. Brooks FJ, Grigsby PW. The effect of small tumor volumes on studies of intratumoral heterogeneity of tracer uptake. J Nucl Med 2013; 55 : 37-42.

31. Hatt M, Majdoub M, Vallières M, et al. 18F-FDG PET uptake characterization through texture analysis: investigating the complementary nature of heterogeneity and functional tumor volume in a multi-cancer site patient cohort. J Nucl Med 2015; 56: 38-44. 